Case Report

\title{
Surgical approach to snus-induced injury of the oral mucosa
}

\author{
Mohammad Kharazmi1), Anders-Petter Carlsson1,2), Pär Hallberg3), Maria Modig1), \\ Lillemor Björnstad1), and Jan-Michael Hirsch²) \\ 1)Department of Oral and Maxillofacial Surgery, Central Hospital, Västerås, Sweden \\ 2)Department of Surgical Sciences, Oral and Maxillofacial Surgery, Uppsala University, Uppsala, Sweden \\ 3)Department of Medical Sciences, Uppsala University, Uppsala, Sweden
}

(Received September 17, 2013; Accepted January 9, 2014)

\begin{abstract}
Snus (Swedish moist snuff) causes lesions in the oral mucosa at the location where pinches are regularly placed. In addition, some patients develop irreversible local gingival recession and sometimes ulcers with perforations to the roots. Such injuries lead to denuded roots that are at risk for caries and periodontal disease, with subsequent esthetic consequences. Therapy for irreversible local gingival recession is currently lacking. In the present report, we describe two cases of successful surgical treatment for irreversible lesions caused by snus.

(J Oral Sci 56, 91-94, 2014)
\end{abstract}

Keywords: snus; moist snuff; smokeless tobacco; snuff dipper's lesion; oral mucosa; gingival.

\section{Introduction}

Snus is the Swedish term for oral snuff, which consists of finely ground moistened tobacco, water, sodium chloride, sodium bicarbonate, humectants such as glycerol and propylene, and natural or artificial flavorings (1). It is addictive and contains carcinogenic tobacco-specific nitrosamines (TSNAs). It is estimated that $21 \%$ of males and $4 \%$ of females in Sweden use snus on a daily basis (2). In recent years, sales of smokeless tobacco marketed as snus have been increasing in the United States (3).

Correspondence to Dr. Mohammad Kharazmi, Department of Oral and Maxillofacial Surgery, Central Hospital, Västerås, SE-721 89 Västerås, Sweden

Fax: +46-21-17-54-30

E-mail: kharazmi.mohammad@gmail.com

doi.org/10.2334/josnusd.56.91

DN/JST.JSTAGE/josnusd/56.91
Snus is marketed loose or portion-packed, and is generally used by placing a 1-2-g pinch in the oral vestibule between the gum and the upper lip. A mucosal lesion, known as snuff dipper's lesion, is always present at the site where a pinch of snus is regularly placed. In general, the lesions are asymptomatic and considered reversible following cessation of snus use (4), although this has recently been questioned (5).

In addition to snuff dipper's lesion, there are also irreversible snus-induced oral mucosal injuries (ISIOMI). Local gingival retraction is the most recognized type of ISIOMI, occurring in approximately one-fifth of users (6). There is a paucity of published literature regarding the management of ISIOMI, and currently no established treatment is available. In this report we describe the successful surgical treatment of two different types of ISIOMI.

\section{Case Reports}

Case 1

A 44-year-old man was referred to the Department of Oral and Maxillofacial Surgery at the Central Hospital, Västerås, for removal of gingival hyperplasia that had persisted for 10 months at the site where the patient had regularly placed snuff quids. At the time of examination the patient had refrained from snuff dipping for ten months. He had been using loose-packed snus for 27 years and had always placed the pinch at the right side of the gingival fold of the upper lip. He did not drink alcohol, and he smoked only occasionally.

On examination, an area of gingival hyperplasia measuring $7 \times 4 \mathrm{~mm}$ was evident at the buccal gingiva between the maxillary incisors (Fig. 1). Severe snusinduced gingival recession was apparent at the first 


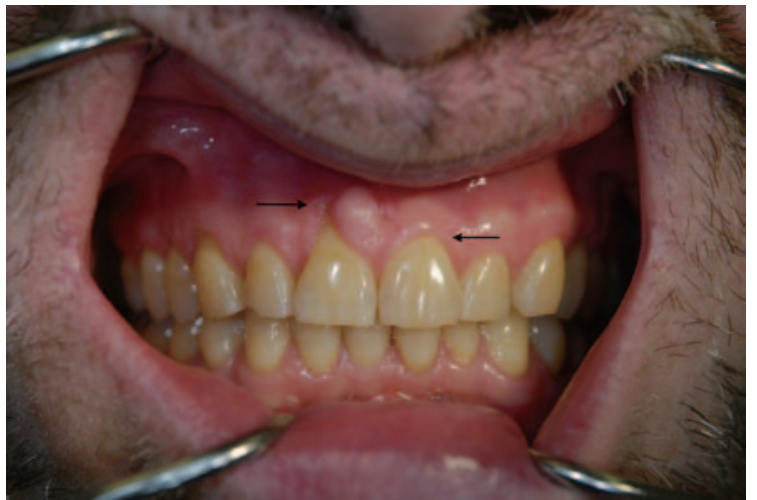

Fig. 1 Severe gingival recession at the first right maxillary incisor and gingival hyperplasia located anterior to the recession 5 months before surgery. Note the highest point of the gingival recession (arrow) in comparison to the normal gingival contour at the first left maxillary incisor (arrow). Case 1.

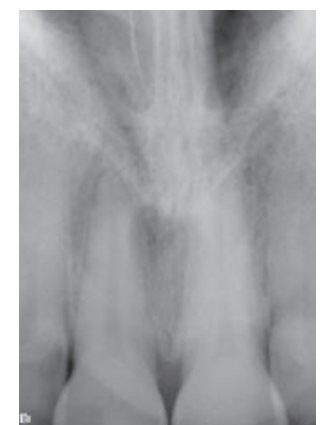

Fig. 2 Radiographic picture of the maxillary incisors. Case 1.

right maxillary incisor (adjacent to the hyperplasia). Apart from gingival recession, the tooth was clinically healthy, and radiographic examination (Fig. 2) revealed no pathology. The patient had been aware of the gingival recession for several years, but had become concerned when it persisted after he had stopped using snus. He requested removal of the hyperplasia and coverage of the root exposure (ISIOMI) for esthetic reasons.

Surgical removal of the altered mucosa was performed 2 months later by raising a full-thickness flap with one horizontal and two vertical incisions (Fig. 3). The horizontal incision was made in the middle of the attached buccal mucosa, and a releasing horizontal incision of the periosteum was performed at the base of the flap to increase mobility. To remove the affected tissue, a vertical complementary anterior incision was made, converging with the first horizontal incision. The flap was advanced laterally and coronally and secured with 4-0 vicryl sutures to enable coverage of the exposed root as well as the site of the mucosal alteration. At 2 weeks of follow-up, uneventful healing was seen. The root of the incisor was covered by firm gingiva and the patient

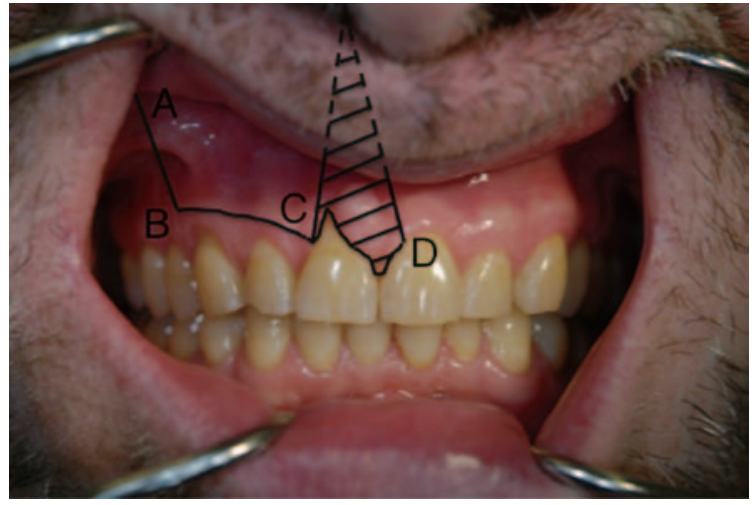

Fig. 3 Laterally sliding and coronally advanced flap. The dashed area is removed. The incision starts at "A" in the mucosa and continues down to half the height of the attached gingiva. The incision then turns horizontally, stretching to point "C". The flap is raised and the periosteum at base of the flap is cut. " $\mathrm{A}$ " is then slid to " $\mathrm{B}$ ", and " $C$ " is slid to D. The flap is then sutured at its new location. Case 1.

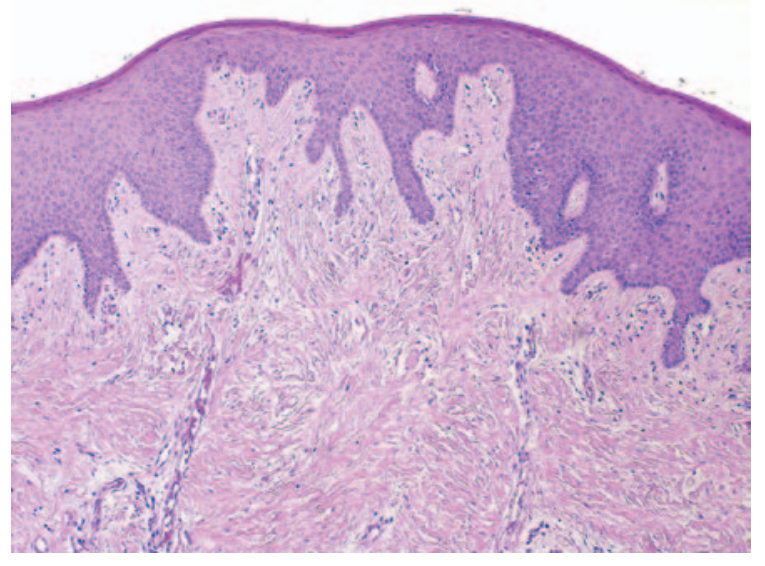

Fig. 4 Histological section showing a representative section of the excised hyperplastic gingiva. The stroma is fibrous and richly vascularized without inflammation. The surface is covered by thin, parakeratinized epithelium. $\mathrm{H}$ $\&$ E stain, original magnification $\times 10$.

reported no discomfort. Histopathological examination confirmed hyperplasia of the gingiva (Fig. 4).

Healthy, firm gingiva displaying a good color match was observed at follow-up 14 months after surgery (Fig. 5). The incisor remained periodontally healthy with a significant reduction of the gingival recession. There was no recurrence of the hyperplasia. The patient reported no discomfort and was satisfied with the outcome.

\section{Case 2}

A 46-year-old man with an unremarkable medical history was referred to the Department of Oral and Maxillofacial Surgery at the Central Hospital, Västerås, because of soreness related to snus-induced mucosal injury of four 


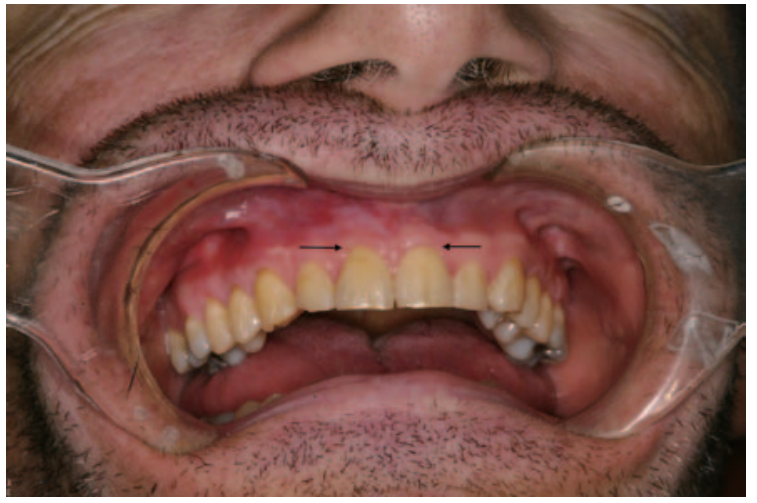

Fig. 5 Postoperative view at 14 months illustrating significant improvement of the gingival recession at the first right maxillary incisor, good color blending, and no recurrence of the mucosal alteration (gingival hyperplasia). Note the highest point of the remaining gingival recession (arrow) in comparison to the normal gingival contour at the first left maxillary incisor (arrow). Compare with the preoperative status (Fig. 1). Case 1.

months' duration. He had been using portion-packed snus for 20 years and had always placed the pinch at exactly the same location as the mucosal fenestration (ulcer) he had developed. He had immediately stopped using snus when the mucosal soreness developed, and he had noticed the injury at the location of pinch placement. He did not drink alcohol and did not smoke.

Examination at four months after cessation of snus revealed a fenestration of the mucosa, measuring approximately $3 \times 1.5 \mathrm{~mm}$, with exposure of the root of the right maxillary canine (Fig. 6). The fenestration was located three millimeters above the cervical gingiva. The exposed root was covered by plaque, and the surrounding mucosa was inflamed and sore. With the exception of the defect described above, the teeth were periodontally healthy. Radiographic examination revealed no pathology (Fig. 7).

The patient was instructed to apply chlorhexidine gel over the lesion for a few weeks, which reduced the local irritation of the mucosa. Surgical treatment was performed with a laterally advanced flap 4 months after the initial examination to obtain mucosal coverage over the exposed root. Overall, the surgery was performed as in case 1, a full-thickness flap being raised with one horizontal and two vertical incisions (Fig. 8). The horizontal incision was made $2 \mathrm{~mm}$ above the cervical gingiva. A releasing horizontal incision of the periosteum was performed at the base of the flap to increase mobility. The flap was laterally advanced and secured with sutures in order to provide coverage of the exposed root. At the 3 -week follow-up point, normal healing was observed.

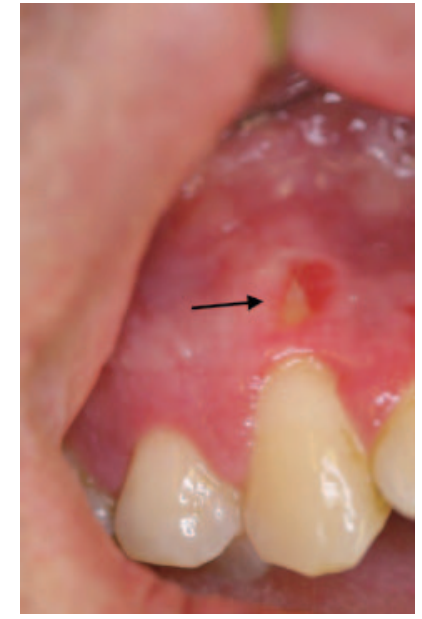

Fig. 6 Fenestration of the mucosa (arrow) and root exposure of the right maxillary canine one week before surgery. Case 2.

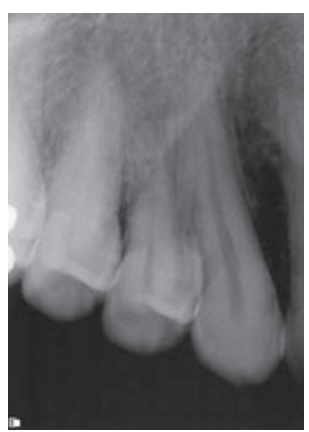

Fig. 7 Radiography of the right maxillary canine. Case 2.

The root of the cuspid was covered with mucosa and the patient reported no soreness or discomfort. Healthy oral mucosa showing a good color match was observed at seven months after surgery, and the cuspid remained periodontally healthy (Fig. 9). The patient reported no discomfort and was satisfied with the outcome.

\section{Discussion}

Both snus-induced mucosal lesions and ISIOMI are less pronounced among users of portion-packed snus than among individuals who use loose snus (4). Among other factors that may affect the severity of lesions and ISIOMI are the placement of the snus (one versus multiple sites) and the total exposure (TE), as estimated by $\mathrm{TE}=\mathrm{A} \times \mathrm{B}$ $\times \mathrm{C}$, where $\mathrm{A}$ is the number of years of snus use, $\mathrm{B}$ the daily duration of use (hours), and $\mathrm{C}$ the amount of snus used daily (grams) $(6,7)$.

Although ISIOMI generally does not require treatment, complications such as discomfort and esthetic disfiguration sometimes lead to discussion about the possibility of surgical correction. Currently, no established corrective surgical therapy is available. As shown in the present two patients, however, successful surgical therapy is possible. 


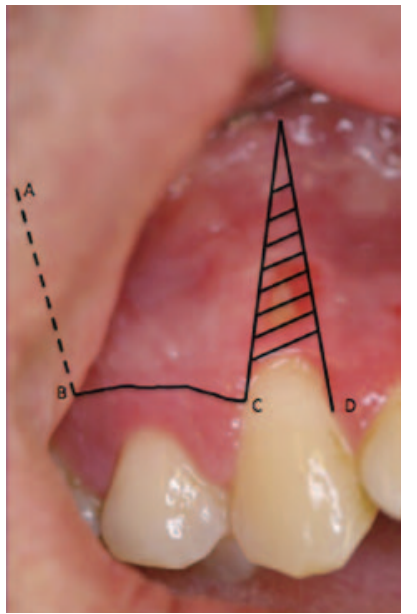

Fig. 8 Laterally sliding flap. The dashed area is removed. The incision starts at " $\mathrm{A}$ " in the mucosa and continues down to half the height of the attached gingiva. The incision then turns horizontally, stretching to point " $\mathrm{C}$ ". The flap is raised and the periosteum at the base of the flap is cut. " $A$ " is then slid to " $\mathrm{B}$ ", and " $\mathrm{C}$ " is slid to D. The flap is then sutured at its new location. Case 2.

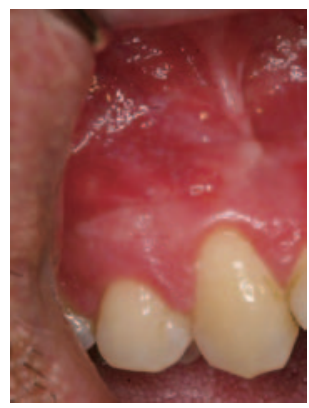

Fig. 9 Postoperative clinical view at seven months showing complete root coverage of the right maxillary canine and good color blending at the site of surgery. Case 2 .

Since snus is the cause of mucosal injury, ongoing use with pinch placement at the location of the ISIOMI should be considered a contraindiction for surgery. Continuous use may jeopardize the outcome of surgery or lead to future recurrence of ISIOMI. Patients should quit using snus six to twelve months prior to surgery for ISIOMI. This period should allow significant recovery of the mucosa and also to some extent confirm that the patient is able to overcome the addiction. Nicotine replacement therapy can be recommended to aid cessation of snus use (8), but should the patient be unable to quit, referral for professional counselling should be considered. For patients who fail to overcome their addiction before surgery, changing to portion-packed snus if using loose snus, and switching the location of pinch placement, is required.

Regardless of the type of ISIOMI, the intended site for surgical treatment must be carefully examined to identify any unfavourable anatomic conditions such as absence of keratinized tissue lateral to the defect or marginal inser- tion of the frenulum. Local application of chlorhexidine gel may reduce gingival inflammation before surgery.

To our knowledge, the present report is the first to suggest a surgical approach for treatment of ISIOMI. However, mucogingival reconstructive surgery is a common procedure for recovery of the original soft tissue morphology in various conditions, and has been proven successful for the treatment of local gingival defects (9).

The surgical procedures described here are not difficult to perform and can best be described as laterally sliding flaps, with or without coronal advancement. The technique may also be described as a modification of the Môczár flap (10). In our experience, the two most important factors for guaranteeing uneventful healing are: refraining from snuff use and a releasing horizontal incision of the periosteum at the base, which is sufficient to allow adequate mobilization of the mucosal flap.

Within the limits of this report, our results show that ISIOMI can be treated successfully by an uncomplicated surgical approach with satisfactory cosmetic results.

\section{References}

1. Cnattingius S, Galanti R, Grafström R, Hergens M, Lambe M, Nyrén O et al. (2005) Health risks of Swedish snus. National Institute of Public Health and Karolinska Institute, Stockholm.

2. Lundquist $\AA$ (2005) Reduced use of tobacco, what is the situation today? National Institute of Public Health, Stockholm.

3. Delnevo CD, Wackowski OA, Giovenco DP, Manderski MT, Hrywna M, Ling PM (2012) Examining market trends in the United States smokeless tobacco use: 2005-2011. Tob Control.

4. Andersson G, Axéll T (1989) Clinical appearance of lesions associated with the use of loose and portion-bag packed Swedish moist snuff: a comparative study. J Oral Pathol Med $18,2-7$.

5. Wallström M, Kjelsberg M, Johannessen AC, Hirsch J-M (2011) The reversibility of snuff-induced lesions: a clinical and histomorphological study. Int J Oral Maxillofac Pathol 2, 4-10.

6. Larsson A, Axéll T, Andersson G (1991) Reversibility of snuff dippers' lesion in Swedish moist snuff users: a clinical and histologic follow-up study. J Oral Pathol Med 20, 258-264.

7. Hirsch JM, Heyden G, Thilander H (1982) A clinical, histomorphological and histochemical study on snuff-induced lesions of varying severity. J Oral Pathol 11, 387-398.

8. Wallström M, Bolinder G, Hassèus B, Hirsch JM (2010) A cessation program for snuff-dippers with long-term, extensive exposure to Swedish moist snuff: a 1-year follow-up study. Acta Odontol Scand 68, 377-384.

9. Kim DM (2011) Periodontal plastic surgery procedures reduce localized gingival recession defects. J Am Dent Assoc 142, 850-851.

10. von Wowern N (1982) Closure of oroantral fistula with buccal flap: Rehrmann versus Môczár. Int J Oral Surg 11, 156-165. 